\title{
Alkoholabhängigkeit - chronische Erkrankung? Vorsicht vor einer pauschalen Klassifikation!
}

\begin{abstract}
Eine aktuelle Studie zeigt die Relevanz des Schweregrades der Alkoholkrankheit für deren Fortbestehen und das Rückfallrisiko. Als Verlaufsmodulatoren gelten auch die Symptome Depression und Angst.
\end{abstract}

$D^{i}$ ie Alkoholabhängigkeit unterliegt in ihrem Verlauf einer Dynamik, die unter anderem von Risikofaktoren aus der Familienanamnese, dem Geschlecht, einer belastenden Kindheit, dem Bildungsstand, der Persönlichkeitsstruktur und potenziellem Nikotinkonsum mitbestimmt wird. In dieser Studie suchten Boschloo et al. prospektiv in einem heterogenen Patientenkollektiv nach unabhängigen Variablen zur Prädiktion des Krankheitsverlaufs nach zwei Jahren.

253 Studienteilnehmer mit remittierter Alkoholabhängigkeit und 135 mit aktuell vorliegender Alkoholkrankheit wurden aus der Allgemeinbevölkerung, in Hausarztpraxen oder in ambulanten psychiatrischen Einrichtungen rekrutiert. Mithilfe verschiedener Messinstrumente wurden der Schweregrad der Alkoholproblematik (AUDIT) im Verlauf des letzten Jahres, depressive (IDS) und ängstliche (BAI) Symptomatik, relevante Persönlichkeitsfaktoren (NEO), das Vorliegen eines Traumas in der Kindheit, negative Lebensereignisse des vergangenen Jahres und das Rauchverhalten sowie der Konsum illegaler Drogen innerhalb des letzten Monats erfasst. Familienanam- nestisch erfragte man Alkohol-, Drogensowie Nikotinkonsum bei Verwandten ersten Grades.

Nach zwei Jahren erlitten 14,6\% der vormals Remittierten ein Rezidiv, 40,7\% der Alkoholabhängigen zeigten eine Erkrankungspersistenz. Die Schwere der Alkoholproblematik zeigte sich als stärkste unabhängige Variable zur Prädiktion eines Rezidivs und der Persistenz der Erkrankung. Die Stärke depressiver und/oder ängstlicher Symptome war mit der Rückfallgefahr assoziiert. Als signifikante Prädiktoren für das Fortbestehen der Erkrankung erwiesen sich neben dem Schweregrad der Erkrankung die Parameter „männliches Geschlecht" und „hoher Bildungsstand“, als prädiktiv für ein Rezidiv stellten sich die Variablen „männliches Geschlecht“ und „Nikotinkonsum“ heraus.

Kommentar: Das Anliegen dieser Studie ist eine frühzeitige Detektion von Alkoholkranken mit Prädisposition für einen ungünstigen Krankheitsverlauf. Allerdings wurden Patienten mit einer schweren Abhängigkeitserkrankung als Erstdiagnose ausgeschlossen. Daher ist die Studie nicht repräsentativ für Patienten

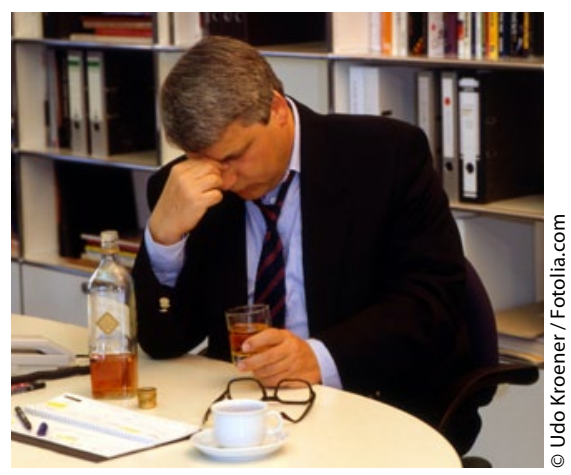

Männer liegen bei Persistenz der Alkoholkrankheit und Rezidiv vorn.

mit schwerer Alkoholabhängigkeit und schafft somit keine reale Abbildung der Rezidiv- und Persistenzraten. Auch waren durch Ausschluss bestimmter Teilnehmer nicht alle Facetten depressiver, psychotischer und ängstlicher Symptomatik berücksichtigt worden. Weitere Forschung ist nötig, um die Effekte dieser spezifischen Störungsbilder auf das Rückfall- beziehungsweise Persistenzrisiko der Alkoholabhängigkeit zu untersuchen. Zusammenfassend motivieren die Ergebnisse der Studie, zusätzlich zur dichotomen Diagnosestellung auch Schweregradindikatoren beim Umgang mit Alkoholkranken im klinischen Alltag einzusetzen. Dies könnte einen entscheidenden Schritt zur Entwicklung individuell angepasster Strategien der Tertiärprophylaxe darstellen. Dr. med. Birgit Braun

Boschloo L, et al. Predictors of the 2-year recurrence and persistence of alcohol dependence. Addiction 2012; 107 (9): 1639-40

\section{Stadien der Alkoholabhängigkeit unterscheiden sich im funktionellen MRT}

\section{Durch Präsentation von Alkoholschlüsselreizen ausgelöste Aktivierungsveränderungen in bestimmten Hirnregionen können einen Hinweis auf die aktuelle Krankheitsphase der Alkoholabhängigkeit geben.}

$\mathrm{D}$ ie Alkoholabhängigkeit ist eine chronische Erkrankung und äußert sich in Craving- und Rückfallphasen. Die Aufmerksamkeit für Alkoholschlüsselreize verändert sich abhängig von diesen Zyklen. Meist geht eine vermehrte Exposition mit alkoholassoziierten Umweltreizen mit einer Steigerung des Alkoholverlangens einher. Daher spielt das reiz- induzierte Craving eine große Rolle in der Aufrechterhaltung der Alkoholabhängigkeit.

In der vorliegenden Studie wurden während einer Aufgabe mit hoher Aufmerksamkeitsanforderung unregelmäßig eingestreute, aufgabenunabhängige alkoholassoziierte sowie erstmals auch nicht alkoholassoziierte Reize dargebo- ten und die Hirnaktivität bei den ablenkenden Alkoholschlüsselreizen mittels fMRI evaluiert. 44 alkoholabhängige $\mathrm{Pa}$ tienten (aktuelle Trinker $n=16$; Kurzzeitabstinente $\mathrm{n}=15$; Langzeitabstinente $\mathrm{n}=13$ ) und 20 Kontrollen ohne Alkoholvorgeschichte wurden eingeschlossen. Im Rahmen eines Event-Related-Designs wurden während drei Runs regelmäßig 